\title{
Juan Carlos Tedesco y el pensamiento educativo: Reflexiones sobre un recorrido intelectual
}

Inés Dussel

\begin{abstract}
Resumen
En este artículo se propone un recorrido por algunos textos publicados por Juan Carlos Tedesco a lo largo de cinco décadas, menos conocidos que su obra consagrada, con la intención de rastrear las transformaciones y desplazamientos en su modo de pensar la educación. A través del análisis de cinco textos, cuatro de ellos publicados en revistas dirigidas a un público intelectual, se busca identificar referencias y lecturas, modos argumentativos y proposiciones, que den cuenta de su original forma de conceptualizar la escuela y las funciones del sistema educativo y de las interlocuciones y problemáticas que construyó en su recorrido político-intelectual.
\end{abstract}

\section{Juan Carlos Tedesco and educational thought: Reflections on an intellectual journey}

\begin{abstract}
In this article I present an overview of some of Juan Carlos Tedesco's published work along five decades, in some cases not too well-known, trying to trace the shifts and displacements in his conceptualization of education. Through an analysis of five texts, four of which were published in intellectual journals, I intend to identify his references and readings as well as his arguments and propositions, in order to account for the original way in which he conceptualized schooling and the social functions of educational systems, and to revisit the dialogues and problematizations that he produced in his political and intellectual journey.
\end{abstract}

Palabras-claves:

Juan Carlos Tedesco, historia de la educación, teoría educativa crítica, ideología educativa, nuevo capitalismo.

\section{Keywords}

Juan Carlos Tedesco, educational history, critical educational theory, educational ideologies, new capitalism. 
1. Sobre la idea del saqueo y las malas lecturas, puede consultarse el trabajo de Sylvia Molloy (1996) sobre Sarmiento.
2. Este breve recorrido puede complementarse con la completa semblanza biográfica que ofrece Darío Pulfer en la revista Historia de la Educación Argentina de la SAHE (Pulfer, 2017).

\section{Introducción}

El 8 de mayo de 2017 falleció en Buenos Aires Juan Carlos Tedesco, uno de los más destacados investigadores y políticos de la educación en América Latina. Homenajearlo es, además de un merecido reconocimiento, una ocasión propicia para revisar sus aportes al pensamiento educativo de las últimas décadas.

En este artículo quiero proponer un recorrido por algunos textos publicados a lo largo de cinco décadas, menos conocidos que su obra consagrada, con la intención de rastrear las transformaciones y desplazamientos en sus modos de pensar la educación en cada etapa, modos que, quiero sostener, contribuyeron a renovar el pensamiento educativo en estos años. Este abordaje busca aportar a la historia intelectual de la pedagogía argentina en la segunda mitad del siglo XX y principios del siglo XXI. Basándome en trabajos como el de Claudio Suasnábar (2004, 2013), pionero en investigar la historia intelectual del campo pedagógico, y en los análisis de Sebastián Gómez (2016a) sobre la historia reciente de los pedagogos críticos argentinos, propongo aquí trazar el trayecto de los planteamientos sobre la educación de Juan Carlos Tedesco a lo largo de cinco décadas.

Cabe hacer una primera aclaración metodológica. El adjetivo "argentina" para referirme a la historia intelectual de ninguna manera debe leerse como una adhesión a las visiones nacionalistas, generalmente esencialistas, sobre las ideas y el pensamiento. Lo nacional no solamente es una construcción histórica y política particular, que coexiste y está tensionada con otras afiliaciones o agrupaciones, sino que siempre está atravesada por redes y referencias que la exceden. En el caso del pensamiento teórico en las ciencias sociales y humanidades, es claro que se lee y discute con autores y marcos de otras latitudes, pero también que se lo hace desde una perspectiva geopolítica particular, en el caso argentino desde "los márgenes", "la periferia", "el sur". Es una lectura excéntrica en muchos casos, que incluye tanto la búsqueda de reconocimiento e identificación con la tradición humanista europea como la libertad y la distancia para "saquear" esa tradición. ${ }^{1}$ Se lee desde una historia política y cultural particular, que distingue a los intelectuales argentinos de otros casos regionales, pero probablemente también se requiera hacer distinciones entre regiones, instituciones y grupos. Esta posición metodológica no niega la existencia de un campo profesional académico y de tradiciones intelectuales que se reconocen en bordes definidos por el Estado nación, pero no quiere dar por sentado que la configuración nacional es la única variable o dimensión a considerar en el estudio de las ideas pedagógicas.

El artículo se organiza en dos partes. En la primera sección, más breve, se recuperan algunos datos de su biografía que permiten contextualizar el análisis que se ofrece en la segunda sección sobre algunos textos producidos a lo largo de cinco décadas de producción teórica sobre la educación. La perspectiva adoptada busca inscribir a Juan Carlos Tedesco en la historia educativa argentina reciente, rastreando su sistema de referencias y lecturas, así como los modos de enunciación y contenidos de sus argumentos, y vinculándolos a los debates intelectuales y políticos de este período.

\section{Algunas notas biográficas ${ }^{2}$}

Formado en Ciencias de la Educación en la Universidad de Buenos Aires en los años sesenta, Tedesco fue protagonista de la renovación epistemológica y político-educativa de esa época, que desplazó la centralidad de la filosofía como fundamento de la teoría educativa hacia las ciencias sociales. Trabajó como estudiante en los programas de extensión universitaria dirigidos por Amanda Toubes, que influyeron en varios pedagogos de esa generación (Adriana Puiggrós entre ellos); se recibió en 1968, en pleno 
onganiato, dando materias libres y con distancia de la vida estudiantil por la intervención militar de las universidades. Fue maestro, egresado de la Normal de San Justo, en escuelas secundarias de La Matanza, Ezeiza, Mataderos y Liniers. En la década de 1960 se formó al calor tanto de los debates políticos, que acompañó con una participación en la izquierda socialista, un breve pasaje por Política Obrera y un acercamiento breve al maoísmo, como de las lecturas sociológicas y de la teoría crítica marxista que circulaba en la Facultad de Filosofía y Letras.

Su primer libro, Educación y sociedad en la Argentina, 1880-1900, publicado originalmente por una pequeña editorial en 1970, pero que pronto se convirtió en un clásico, fue prologado por Gregorio Weinberg, titular hasta el golpe de 1966 de la cátedra de Historia de la Educación Argentina en la UBA. Este libro signó un modo renovado de entender la educación a la luz de las nuevas teorías sociológicas y económicas. En debate con las teorías del capital humano y la historia tradicionalista de Manuel Solari, pero también con las teorías marxistas reproductivistas, Tedesco afirmó que la educación cumplió una función política de formación de los ciudadanos en América Latina. Su preocupación en ese y otros trabajos fue comprender la especificidad de lo educativo, sin perder de vista su relación con la política y la sociedad, pero sin reducirla a las dinámicas externas. Su condición de maestro y pedagogo lo llevaron a interesarse tanto en las leyes educativas y los cambios socioeconómicos como en la didáctica de la lectoescritura y las condiciones de trabajo de los docentes. A ese libro pionero siguieron otros, como Educación y clases sociales (1973), y sobre todo una serie de artículos que sentaron posición sobre su lectura de los vínculos entre la educación, la sociedad, la política y la economía, como se verá en la sección siguiente.

Esta producción fue realizada en el marco de diversas adscripciones institucionales, que constituyeron condiciones de enunciación importantes para sus planteos educativos. A su temprana actividad universitaria en las Universidades de La Plata, La Pampa y Comahue en Argentina, pronto se sumó su trabajo en los equipos de la Unesco, el Centro Regional de Educación Superior en América Latina y el Caribe (CRESALC) y la Oficina Regional de Educación para América Latina y el Caribe (OREALC). En la Cepal y con apoyo de la Unesco, creó junto con Germán Rama el programa Desarrollo y Educación en América Latina y el Caribe, que entre 1976 y 1982 elaboró los diagnósticos y propuestas que sentarían las bases de las políticas educativas en las recuperadas democracias del Cono Sur en la década de 1980. Estuvo en el grupo fundador del área Educación de FLACSO-Argentina, y allí creó la primera maestría con orientación educativa en ese país junto a un grupo de intelectuales que, como él, tendrían destacada participación en la política educativa de las décadas siguientes, entre ellos Cecilia Braslavsky y Daniel Filmus, que fueron colegas cercanos en afecto y afinidad intelectual.

Hacia el final de la dictadura, Tedesco se mudó a Caracas para desempeñarse al frente de la CRESALC, donde inició una experiencia de más de dos décadas dentro de organismos internacionales. En 1986 volvió a mudarse, esta vez a Santiago de Chile para dirigir la OREALC; en 1992 se trasladó a Ginebra, donde fue director de la Oficina Internacional de Educación (BIE) de la Unesco. Entre 1997 y 2005 actuó como director de la Oficina Regional del IIPE-Unesco en Buenos Aires.

Desde 2005 hasta 2011 estuvo en los puestos más altos de la política educativa argentina, primero como Secretario de Educación y luego como Ministro de Educación de la Nación, y en una tercera etapa como encargado de la planeación estratégica educativa; su trabajo de coordinación y redacción fue central para la promulgación de la Ley de Educación Nacional de ese país en 2006. Durante sus últimos años estuvo al frente del Programa para la Mejora de la Enseñanza de la Universidad Nacional de San Martín. Publicó en ese tiempo otros trabajos significativos, como los libros Educación y justicia 
3. Sigo aquí la postura del historiador de las ideas Lucien Jaume: "La verdadera pregunta no es: “¿Tuvo Rousseau alguna influencia en los jacobinos?', puesto que es una pregunta sin respuesta y, además, compara cosas que no son comparables. La pregunta podría ser: ‘¿por qué algunos jacobinos citan a Rousseau?'. Debemos plantearnos, por tanto, en qué contexto y con qué fines" (Jaume, 2004: 118).

4. La totalidad de los números están disponibles gracias al trabajo de Diego Cousido (cfr. http://www.ahira. com.ar/libros.php). Puede consultarse también el trabajo de Sebastián Gómez sobre esta revista (2016b).
5. El editorial del número 31, presumiblemente escrito por el consejo de redacción, después de un diagnóstico general ("Crisis es, sin duda, la palabra que aparece casi inmediatamente si se quiere definir hoy el problema de la escuela en la Argentina"), hace referencia a los debates del Congreso Docente de Huerta Grande, donde al menos un tercio del movimiento gremial docente se alineó con una alternativa de transformación radical del sistema educativo, "cuya realización práctica comporta la subversión revolucionaria de la sociedad argentina" (Los Libros,1973: 3). El clima era de abierta radicalización política, y la crítica a la opresión escolar estaba difundida más allá del terreno académico-universitario. social en América latina (2012) y La educación argentina hoy (2015), que evidenciaron la continuidad de su interés por las políticas y las prácticas educativas.

\section{Tedesco y las teorías de la educación: lecturas, claves, anclajes}

¿Cuál es el aporte original de Juan Carlos Tedesco para pensar la educación y, particularmente, la escuela? En esta sección, quisiera rastrear algunos textos clave que publicó en revistas intelectuales argentinas y latinoamericanas (Los Libros, Nueva Sociedad, Punto de Vista), que marcan su visión teórica y política sobre el sistema escolar y la pedagogía, a los que se agrega un trabajo editado en un libro de circulación más restringida que su obra consagrada, en gran parte debido a su inscripción en condiciones de represión y clandestinidad (Biasutto[Tedesco], 1977; Tedesco, 1973, 1975, 1986, 2004). Son textos formulados para el debate con otros intelectuales, y expresan su recorrido como pensador que dialoga con las ciencias sociales y que aporta lecturas e hipótesis de largo alcance sobre la educación argentina. En esta aproximación, me interesa reconstruir sus argumentos y la red de lecturas de la que surgieron sus ideas: ¿a quién citaba Tedesco en cada una de las etapas, y con qué fines? ${ }^{3}$ ¿Qué sistemas de referencias usó para construir sus enunciados pedagógicos? ¿Con qué disciplinas y tradiciones se ligaba, y cómo? ¿Qué efectos tuvieron estos sistemas de citas y estos diálogos en su reflexión sobre la educación?

\subsection{La ideología de la educación en la revista Los Libros (1973-1975)}

El análisis comienza con dos textos publicados en 1973 y 1975 en la revista Los Libros, una publicación independiente dirigida inicialmente por Héctor Schmucler con el modelo de la revista francesa La Quinzaine Littéraire. ${ }^{4}$ Los artículos de Tedesco se publican luego de la fractura de la revista en el número 29, que lleva a la partida de Schmucler y otros miembros del consejo de dirección y a la organización de un consejo de dirección conformado por Ricardo Piglia, Beatriz Sarlo y Carlos Altamirano, en ese momento con orientación maoísta. Los artículos son "Educación e ideología en Argentina. Notas para una investigación” (1973) y "Educación y política en América Latina” (1975).

Los títulos son en sí mismos elocuentes. Los textos quieren producir una reflexión teórica doblemente situada: geopolíticamente, tanto en la Argentina como en América Latina, y epistemológicamente, en el cruce entre investigación y política. En el primer artículo, publicado en agosto-septiembre de 1973, en plena efervescencia política, se plantea la utilidad del concepto de ideología para analizar tanto las prácticas y textos de la educación como las teorías. Dice el autor, casi al comienzo del artículo:

\begin{abstract}
Ya no se trata solo de aprender a leer, sino de aprender también a defenderse de cierto tipo de lectura; no se trata solo de tener acceso a la escuela, sino de defenderse contra los rituales opresores de una práctica escolar que, bajo una aparente atmósfera de neutralidad y de sacralismo, introduce los contenidos y las conductas que perpetúan el sistema social vigente. (Tedesco, 1973: 4)
\end{abstract}

La ilusión tecnocrática de que la escuela sirve a todos por igual está rota, y lo que queda es aguzar la crítica y desarrollar otras propuestas (cfr. Gómez y Kaplan, 2017). En el señalamiento de cómo la ideología configura las prácticas educativas, Tedesco (1973: 5) plantea la necesidad de adoptar una perspectiva antropológica, "poco o nada explotada en este ámbito", y de describir la realidad argentina con perspectiva histórica. Podría decirse que en sus trabajos posteriores asumió la perspectiva del historiador y del sociólogo más que la de antropólogo de la educación, pero es destacable que en este 
momento tan particular de la política argentina Tedesco plantee la necesidad de tomar esa tradición disciplinaria para enriquecer el análisis de los fenómenos educativos. Los autores que se citan en este trabajo son Bourdieu y Passeron (La Reproducción en su edición francesa de 1970) y Althusser (Ideología y Aparatos Ideológicos del Estado, editado por Nueva Visión ese mismo año, 1973, pero que se consigna como "mimeog", ¿quizás leyó pruebas de galera?); en el artículo también se recomienda ver "en la literatura latinoamericana" a Tomás Vasconi (su artículo "Contra la escuela", publicada en la Revista de Ciencias de la Educación dirigida por el propio Tedesco, en el mismo año), y una referencia genérica a los trabajos de Iván Illich y Paulo Freire, algo que sorprende debido a su posterior distanciamiento de sus perspectivas.

La marca althusseriana es visible en su consideración de los textos escolares como prácticas ideológicas, a los que distingue de las prácticas concretas del cotidiano escolar. Tedesco destaca la posibilidad de prácticas "sustancialmente distintas de las que vivimos cotidianamente" (1973: 5), como puede verse en las experiencias relatadas en Cartas a una Profesora de los alumnos de Barbiana ${ }^{6}$ o en el libro Vida de un maestro de Jesualdo. Ofrece un análisis ideológico de los libros de texto de la primera mitad del siglo XX elaborado junto a su cátedra de Historia de la Educación Argentina y Americana de la UNLP, que revisa desde obras de J. J. Berrutti y P. Pizzurno hasta los textos del primer peronismo. En esta revisión, el nudo conflictivo es el análisis del fenómeno peronista, para el que Tedesco refiere a Murmis y Portantiero (Estudios sobre los orígenes del peronismo, editado en 1971), y a Emilio de Ípola (en un texto mimeografiado, Análisis de Ideologías, editado en 1971 por FLACSO-Chile), los dos últimos, parte del grupo Pasado y Presente, un grupo marxista cordobés de producción de pensamiento crítico. Apoyado en estas lecturas, para Tedesco la clave es comprender al peronismo dentro de la lucha de clases y analizar la alianza de clases que este movimiento expresa, lo que permite entender sus contradicciones, por ejemplo, entre la dominación política y la dominación ideológica (nombrada explícitamente como "los sectores clericalestradicionales") (1973: 11). Tedesco señala que los libros de texto peronistas muestran una expresión concreta de cambio en la mención a la nacionalización de las empresas y la aparición de los obreros como personajes históricos, pero no deja de señalar críticamente el protagonismo de Perón y Evita para referirse a "las conquistas de los sectores populares" (ibíd.: 6). Cita también textos de J. Silber y R. Roncagliolo incluidos en la Revista de Ciencias de la Educación que él dirige.

A nivel de la teoría pedagógica y sus vínculos con la ideología, Tedesco analiza la historia de las ideas pedagógicas desde el positivismo al primer peronismo; no provee otras claves de interpretación que las esbozadas en sus artículos sobre el directivismo y espontaneísmo, o las presentes en su libro de 1970, Educación y sociedad en Argentina, 1880-1900. Pero avanza algunas hipótesis sobre la debilidad del peronismo de ir más allá de la vaga formulación de una "pedagogía nacional", crítica que seguramente alcanzaba al peronismo de 1973 y no solamente al primer peronismo. Al mismo tiempo, destaca logros de la ideología peronista en las prácticas educativas del período 1945-1955: la expansión de la enseñanza técnico-profesional y la desaparición de "cualquier intento de desescolarización" (Tedesco, 1973: 11) -una expresión que, a la par que reconoce el crecimiento de la matrícula escolar en esa etapa, evoca negativamente a Illich, evidencia de su distancia con el discurso contraescolar-.

En el artículo que se publica un año y medio después, "Educación y política en América Latina" (marzo-abril 1975), en la misma revista, pero ya con otro consejo editorial, Tedesco aborda de manera directa la cuestión del peronismo como populismo. Las citas incluyen varios de los trabajos sociológicos y políticos de la época: al texto de Germani, Di Tella y Ianni (Populismo y contradicciones de clase en América Latina, editado por Era en México en 1973), se le suman los trabajos de Francisco Weffort sobre Brasil (1968) y -citada de manera crítica- la compilación de Ionescu y Gellner sobre populismo editada
6. Este trabajo había sido discutido en un texto publicado en un número anterior de Los Libros, escrito por Justa Ezpeleta, Marta Teobaldo y Guillermo García (Ezpeleta et al., 1970).
7. El editorial de este número (40) es organizada en dos columnas, una firmada por Piglia y otra por Sarlo y Altamirano, en las que se exponen las disidencias y Piglia anuncia su renuncia a la revista. 
por Amorrortu en 1970. Aparecen también varias referencias a la teoría dependentista: se cita explícitamente a Fernando H. Cardoso y Enzo Faletto, Osvaldo Sunkel y Pedro Paz, Ruy Mauro Marini, Theotônio dos Santos, Amílcar Herrera, Oscar Varsavsky y Darcy Ribeiro. La perspectiva de análisis que se propone es latinoamericana, pero los datos estadísticos y argumentos se refieren mayoritariamente al contexto argentino.

En este texto, puede verse que, conforme al proceso de radicalización y fractura del peronismo, la lectura del peronismo es más crítica que en el artículo anterior. Sin desconocer los logros en la ampliación de la matrícula y la posibilidad de una creación industrial autónoma por la sustitución de importaciones, Tedesco afirma que "el populismo representa el momento en el cual los sistemas educativos asumen su carácter de aparatos ideológicos de estado en forma más ostensible" (1975: 13). Es difícil no leer este texto sin pensar en el aumento de la represión y la radicalización de los sectores de izquierda; la crítica al peronismo muestra menos concesiones que las de 1973.

Sin embargo, en la segunda parte del artículo se despliegan argumentos sociológicos sobre la enseñanza técnica que se fundamentan en trabajos de Germán Rama y de Aldo Solari publicados en la revista de FLACSO-Chile en 1971 y 1972, y también en argumentos de Tomás Vasconi e Inés Reca que identifican contradicciones sobre el rol de las clases medias educadas en los países latinoamericanos, referencias que ofrecen lecturas más matizadas y ambivalentes respecto a la eficacia de la dominación ideológica y al carácter de mero "aparato ideológico" del sistema educativo, y de la acción del peronismo a nivel educativo. Aparecen también referencias a las dictaduras tecnocrático-militares y a la penetración imperialista que, en su proceso de concentración monopólica y exclusión social y política, llevan a la radicalización de los jóvenes (Tedesco, 1975: 16). Los párrafos finales son significativos: en ellos se denuncia el vaciamiento de contenidos de la educación media. Según el análisis de Tedesco, su función ideológica no es ya la formación política de la clase dirigente ni de las clases medias; tampoco es divulgar una ideología neutra cientificista. Citando a Telma Barreiro y su denuncia de los mecanismos ocultos de la alienación educativa, Tedesco concluye que...

si bien la "des-ideologización" intentada desde la perspectiva cientificista-tecnocrática no pudo concretarse, ella se logró a través, precisamente, de un largo proceso en el cual la enseñanza media fue quedando totalmente vacía de contenidos que tuvieran algo que ver con la realidad y donde las fórmulas metodológicas más frecuentes resultan incapaces de generar un desarrollo mínimamente creativo de la inteligencia. (Tedesco, 1975: 16)

La rigidez y el anacronismo de la enseñanza media tendrían, entonces, una función ideológica más compleja que la reproducción de la ideología dominante: su tarea es excluir por aburrimiento, por sobrecarga pedagógica, por irrelevancia. Tedesco afirma en este momento un diagnóstico sobre el vaciamiento de la escuela de contenidos relevantes que mantendrá en trabajos posteriores, aunque en esos nuevos trabajos adopte lenguajes menos explícitos y contundentes sobre la participación del sistema educativo en las relaciones de dominación social que en esta primera etapa.

\subsection{La educación desde la clase obrera: una perspectiva gramsciana}

8. Según testimonios informales que es necesario confirmar, Tedesco era el asesor educativo de Schavelzon en esos años. El vínculo probablemente provenía de los intentos de publicación de la Revista de Ciencias de la Educación en la editorial Galerna, dirigida entonces por Schavelzon (Gómez y Kaplan, 2017). Galerna

Durante la dictadura Tedesco permaneció en Buenos Aires, donde se encargó del programa sobre empleo y educación en el PNUD (Pulfer, 2017: 10). En 1977 publica en México un libro, Educación y Clase Obrera, bajo el seudónimo Carlos Biasutto, entonces arquero de Atlanta (club donde había trabajado), en la editorial Nueva Imagen. Cabe señalar que Nueva Imagen estaba dirigida por un argentino, Guillermo Schavelzon, y tuvo una colección educativa donde se incluyeron obras de Tomás Vasconi, Adriana Puiggrós y algunos marxistas italianos. ${ }^{8}$ El libro es una compilación de artículos en la 
que se incluyen textos de Herbert Gintis (coautor, con Samuel Bowles, del influyente Schooling in Capitalist America de 1971), W. Norton Grubb y Marvin Lazerson, dos economistas de la educación críticos, y cuatro contribuciones de pensadores italianos: Ettore Gelpi, Antoni Santoni Rugiu, Emilio Samek Ludovici, M. C. Costa y Aldo Visalberghi, provenientes de la revista italiana Scuola e Città, un órgano de pensamiento pedagógico laico fundado en la posguerra.

En este texto, Tedesco propone una lectura de la problemática de la educación para el trabajo y la perspectiva obrera. Desde el título, Tedesco deja en claro que se quiere ocupar de los vínculos de la clase obrera con la educación. Esos vínculos refieren tanto a la formación laboral como a la mirada hacia la escuela desde el punto de vista de los intereses de la clase obrera; el lenguaje marxista está muy presente. Su introducción, en la que no hay más citas que a los autores incluidos en la compilación, muestra argumentos pedagógicos de mayor complejidad que los de 1973, aunque también evidencia continuidades en los enunciados. Biasutto[Tedesco] afirma en 1977 que el vínculo entre las relaciones sociales en la escuela y en el trabajo no es unívoco, ni hay una correlación perfecta:

[L]a presión planteada por las exigencias propias de la teoría pedagógica que, por lo menos desde Pestalozzi en adelante, viene postulando la necesidad que el vínculo maestro-alumno se democratice en todos los sentidos posibles. Si bien la vigencia de los principios teóricos de la pedagogía es muy relativa (la disociación entre teoría pedagógica y práctica educativa es uno de los temas menos estudiados en teoría de la educación), existe una tensión permanente que, en determinadas circunstancias históricas, permite a ciertos sectores del aparato escolar actuar bajo el predominio de modelos de relaciones sociales antagónicos o, al menos, diferentes a los vigentes en la estructura social. (Tedesco, 1977: 13)

En este texto se dice, más claramente que antes, que la escuela es un espacio de lucha social, lo que impide que los objetivos de los sectores dominantes tengan una eficacia completa. Si se compara esta formulación con la del artículo de 1973, cuando se hablaba de la posibilidad de prácticas "sustancialmente distintas de las que vivimos cotidianamente" (Tedesco, 1973: 5), se ve en la reflexión sobre la formación obrera una mayor claridad en identificar las tensiones y contradicciones de la escuela, y en darles un basamento teórico que supera el mero reconocimiento de experiencias alternativas como las de Jesualdo o los maestros de Barbiana. Contra la crítica althusseriana, Biasutto[Tedesco] afirma que la escuela es "uno de los ámbitos más susceptibles de presión por parte de los intereses antagónicos al sistema de dominación capitalista" (1977: 14). La perspectiva es matizada, y se perciben ecos de los gramscianos italianos en sus argumentos. Tedesco plantea que la democratización de la enseñanza encuentra tres límites: el carácter dual del sistema educativo (la reproducción de la dicotomización de las jerarquías sociales); los contenidos de la enseñanza (tanto los elementos cognitivos como los no cognitivos del proceso de aprendizaje que producen resultados desiguales); y el acceso al trabajo (la relación entre la educación y las posibilidades de ejercer un tipo de actividad que permita realmente la expresión de las capacidades y potencialidades desarrolladas por el proceso educativo) (ibíd.: 15). Pero esos tres ejes son precisamente las líneas desde los cuales puede diseñarse una estrategia educativa "desde el punto de vista de los intereses del movimiento obrero" (ibíd.), estrategia que debe luchar por una nueva organización institucional (la educación integral), un nuevo currículum que renueve los contenidos, y una nueva organización del trabajo, todos considerados como "bloque único de problemas" (una idea que retoma de Santoni Rugiu).

Sorprende leer, en esta introducción, el llamado de Tedesco a "ir organizando desde ahora al proletariado como clase dirigente" (ibíd.), y no esperar al momento de la 
9. Es sabido que Juan Carlos Tedesco fue especialmente solidario y generoso en los años oscuros de la Argentina; escondió en su casa junto con Nilda, su mujer, a varios de sus amigos pedagogos perseguidos por los militares, y los ayudó a escaparse. revolución. Hay un optimismo inesperado en medio de la época más sangrienta de la dictadura argentina, pero es probable que Tedesco tuviera en mente lectores de otras latitudes (México, Italia), y que eso lo ayudara a pensar en coyunturas distintas a las que se vivían en Buenos Aires. ${ }^{9}$ Afirma que las propuestas pedagógicas progresistas deben buscar definir "espacios institucionales aptos para que la clase obrera vaya ejerciendo efectivamente sus derechos y desarrollando sus capacidades" (ibíd.). Desde su perspectiva, esas propuestas tienen que ser serias y rigurosas; aun en aquellas propuestas comprometidas "con una tarea 'revolucionaria " se ve esta "falta de rigor y sistematización" que les impide dejar su posición subordinada (ibíd.: 17). El "movimiento obrero internacional" (ibíd.) debe definir una estrategia curricular y metodológica que luche contra la devaluación de la enseñanza.

Se fortalece, en este texto de 1977, una perspectiva pedagógica más específica sobre la educación y lo escolar, que ofrece también mayor optimismo en el largo plazo. Biasutto [Tedesco] adopta un lenguaje distinto al de 1973/1975, provisto en buena parte por los pedagogos italianos: la renovación curricular, la reorganización institucional, el replanteo de las formas de trabajo y del vínculo con el mundo del trabajo. Después de la radicalización, parece haber un repliegue a problemas de larga data, que conectan con su trabajo de historiador y también de maestro normalista. Pero aparece también una línea hacia el futuro, una voluntad programática y de construcción que en los años siguientes cobrará todavía más fuerza, como puede verse en los textos que se analizan a continuación.

\subsection{El futuro de la educación, 1986-2004: un llamado a los intelectuales}

10. Antes de salir de Argentina, Tedesco había escrito, junto a Cecilia Braslavsky y Ricardo Carciofi, El Proyecto Educativo Autoritario (1982) donde sentaron las bases del análisis político-educativo de la dictadura. Tedesco describió algunos de los debates y dilemas de esos años en el texto homenaje a Cecilia Braslavsky (Tedesco, 2016).

11. Para un análisis de la historia de la publicación en esos años, puede consultarse el trabajo de Aranguren (2010).

12. Puede compararse estos enunciados con la "fuerte entonación antiestatalista" que describe Rinesi (2015) como típica de la posdictadura argentina. En este aspecto, Tedesco parece permanecer al margen, pensándose más bien como hombre de Estado.
La caída de la dictadura encuentra a Tedesco en Caracas y luego en Chile. ${ }^{10}$ Como director de la OREALC-Unesco en Santiago de Chile, publica en la revista Nueva Sociedad, entonces editada en Caracas por la Fundación Friedrich Ebert, de la socialdemocracia alemana, ${ }^{11}$ un artículo que plantea una lectura de conjunto de la educación en las posdictaduras. El título, "Crisis económica, educación y futuro en América Latina", incluye la cuestión de la prospectiva, de manera similar a otro texto que Tedesco publica en 2004 en la revista argentina Punto de Vista, dirigida por Beatriz Sarlo, "Incertidumbre y esperanza. Sobre la educación y el futuro". Ambos artículos, aunque separados por casi veinte años, permiten ver algunos temas clave de la interlocución que Tedesco propone al campo intelectual, y del lugar que otorga a la educación en la producción de otros futuros.

El texto de Nueva Sociedad puede considerarse como ilustrativo del período cepalino de Tedesco. Las citas del artículo remiten a un espacio político-académico de las ciencias sociales críticas, pero ya no marxistas ni radicalizadas: Torcuato Di Tella, Ernesto Ottone, Guillermo O’Donnell, Victor Tokman, José Joaquín Brunner, Raúl Prebisch. La mayor parte de las publicaciones que cita son de la revista de la Cepal o de FLACSO-Chile. Buena parte de los argumentos son socioeconómicos, y refieren a los desafíos de desarrollo económico de los países latinoamericanos en un contexto de crisis internacional, transformaciones tecnológicas y cambios en la división internacional del trabajo. El argumento es, en muchos casos, economicista: es mayormente a partir de las necesidades del desarrollo económico que se derivan las funciones y tareas de la educación. Pero hay también claves políticas respecto a la consolidación de las democracias, el fortalecimiento de los Estados nación y la superación de la debilidad consuetudinaria de los actores de la sociedad civil, y también del propio Estado. Dice el autor: "el futuro plantea el desafío de desarrollar fórmulas de nacionalismo económico y político, tradicionalmente patrimonio de regímenes militares, en un marco político de democracia representativa" (Tedesco, 1986: 82).

La enunciación es estatalista: el sujeto de las políticas es el Estado nación, y el lugar de los intelectuales es contribuir desde dentro a formular y desarrollar las políticas. ${ }^{12} \mathrm{La}$ 
audiencia imaginaria parecen ser los intelectuales convertidos en analistas simbólicos, al decir de José Joaquín Brunner, que van a intervenir en las políticas de Estado en esas décadas. Tedesco plantea que hay que pensar en alternativas regionales y no solamente nacionales, que contribuyan a mayores grados de autonomía económica. La educación, en este contexto, tiene varias funciones que cumplir. Por un lado, si los modelos de desarrollo económico del futuro serán intensivos en conocimiento, el sistema educativo tiene que preocuparse por formar una base común científico-técnica, una suerte de alfabetización científica masiva. Las políticas educativas también tienen que ocuparse del sistema de investigación científico-tecnológico, para aportar a la industria recursos y conocimientos adecuados a los desafíos de su transformación.

Sin embargo, pese al peso de la argumentación socioeconómica en las referencias y lecturas, la función política (aquella que Tedesco identificaba en la oligarquía argentina de fines del siglo XIX) también cobra importancia. El argumento del texto es que, en sociedades crecientemente desarticuladas, tanto por la herencia traumática de las dictaduras como por la expulsión de parte de la población a la marginalidad y el desempleo, es importante generar formas de solidaridad entre los distintos sectores sociales. Aun cuando sostuvo algunos de los ejes del escrito gramsciano de 1977 en relación a renovar las propuestas curriculares, institucionales y las formas de trabajo, Tedesco parece haberse desplazado de Gramsci a Durkheim en su defensa de la educación como cemento social de la sociedad:

\begin{abstract}
Las estrategias de desarrollo futuro suponen la definición de una nueva constelación de valores acerca del desarrollo: la solidaridad, la eficiencia, la participación y la creatividad serían, en consecuencia, algunos de los valores que estarían asociados a las estrategias de desarrollo y en cuya difusión -a través de la socialización escolar-el sistema educativo tendría un significativo papel a cumplir. (Tedesco, 1986: 86)
\end{abstract}

Sin embargo, Tedesco no prevé, como Durkheim, una integración armoniosa, ni confina los conflictos a la anomia social; en eso, sostiene su tributo a formas de pensamiento crítico que ven a las contradicciones y conflictividades como parte de lo social. La última sección del artículo está dedicada a discutir alternativas concretas de reforma: ¿educación universal masiva o inversión en la formación de la cúspide? ¿Sistema homogéneo o sistema dual? ¿Currículum universalista o adaptado a las demandas y conocimientos locales? El autor abre el abanico de posiciones y se esfuerza en mostrar que cada opción conlleva riesgos y exclusiones. Aboga por tomar el principio de la "heterogeneidad en el punto de partida del proceso de aprendizaje, pero homogeneidad en el punto de llegada" (ibíd.: 90), una idea que puede haber tomado de Dermeval Saviani o de Cecilia Braslavsky, cuyos argumentos sobre la unidad y diferenciación del sistema educativo (1982) retoma, aunque sin citarla. Cabe notar, por otra parte, que Tedesco no incluye en este texto ninguna referencia a estudios educativos.

La discusión sobre el currículum es muy sugestiva, y recupera algunos de los argumentos ya mencionados en los textos de 1975 y 1977, aunque avanza en otras direcciones. Se diferencia de la crítica illichiana y althusseriana de la escuela al mostrar que la escuela latinoamericana reproduce excluyendo y no integrando; menciona también que la imposición ideológica es distinta a la francesa, y pasa por el vaciamiento de contenidos y la expulsión y el fracaso escolar. Frente a esta situación, defiende los conocimientos socialmente significativos que la escuela distribuye como indispensables para participar activamente de la transformación de la sociedad. Si bien reconoce que estos conocimientos pueden implicar una "imposición cultural" y que los saberes científico-técnicos suponen la "ruptura de los desarrollos culturales endógenos" (1986: 89), expresiones que muestran su paso por organismos regionales donde las demandas del plurilingüismo y la interculturalidad son notorias, Tedesco cree que la alternativa no puede ser el aislamiento ni la reproducción de la distribución desigual de los saberes. 
Propone entonces la definición de "un núcleo común de contenidos curriculares" (ibíd.: 90) que tenga en cuenta la heterogeneidad cultural de América Latina, una propuesta que se llevará adelante como política curricular en la década siguiente. En el cierre de su artículo, señala que la dificultad de consensuar ese núcleo de conocimientos básicos comunes es social y no centralmente pedagógica. El futuro que se dibuja desde esta intervención de Tedesco es pactista, consensualista: la apelación a los intelectuales es que trabajen en la dirección de crear los acuerdos y las formas de solidaridad para que la escuela produzca nuevos ciudadanos productivos y sociedades mejor integradas. Aunque la criticidad se sostiene como un valor, pierde la centralidad que tenía en los artículos de la década de 1970 .

Si el futuro que Tedesco avizora en el texto de 1986 tiene algunos nubarrones, el artículo de Punto de Vista de 2004 plantea un porvenir definitivamente más aciago, dominado por la manipulación genética en manos de las corporaciones transnacionales y el declive de los estados nacionales. El artículo evidencia que el lugar de enunciación estatal se ha debilitado considerablemente; pero como en 1986, Tedesco otorga a los intelectuales un rol central en la formulación de un nuevo discurso público que legitime a la educación.

El título, "Incertidumbre y esperanza. Sobre la educación y el futuro", muestra el desplazamiento de las preocupaciones del desarrollo económico e institucional latinoamericano hacia sentimientos y emociones sobre el porvenir de la especie humana. Esto es consistente con los modos de dominación del nuevo capitalismo, en el que se dan paralelamente fenómenos de alta concentración corporativa y extrema atomización individual. Las lecturas y referencias del texto se desplazan hacia el norte: Habermas (El porvenir de la naturaleza humana, edición francesa del 2002), Beck (La sociedad del riesgo global, 2001), Fukuyama (Our Posthuman Future, 2002), Rifkin (The Biotech century, 1999), Thurow (Inventando riqueza, 1999), Fitoussi y Rosanvallon (La nueva era de las desigualdades, edición francesa de 1996), la teoría de la justicia de John Rawls y la crítica a la metamorfosis liberal de Lipovetsky (en su edición francesa de 2002).

El único texto educativo citado es el Informe Delors de la Unesco (La educación encierra un tesoro, 1996), del que Tedesco destaca dos de los cuatro pilares educativos: aprender a aprender y aprender a vivir juntos. Tedesco considera que el nuevo capitalismo no apela a la solidaridad orgánica, porque no le interesa la hegemonía, sino el dominio y la intensificación de la productividad; la solidaridad se vuelve entonces, como ya señaló en 1986, aunque en otras circunstancias, una tarea del sistema educativo. El riesgo mayor es, para Tedesco, el anestesiamiento moral de la población frente a márgenes cada vez menores de decisión sobre la propia vida -como se ve con la manipulación genética-, y una incapacidad creciente de los estados y la política para dar respuestas a problemas que están ya fuera de sus manos.

Frente a esta situación, Tedesco aboga por una educación humanista "que supere la idea de una moral sin base científica y de un desarrollo científico sin control moral" (2004: 20). Como en 1986, los contenidos educativos deben pasar por la formación científica, pero también deben incluir, en línea con el informe Delors, el aprendizaje del valor de la solidaridad orgánica, del vivir juntos, de una sociedad incluyente que conoce y teme los costos de una alta exclusión social. Tedesco insiste en esta última etapa en los valores, con algunas resonancias que pueden parecer conservadoras, pero que parten de su diagnóstico de una crisis política sin precedentes. ${ }^{13}$

En la última sección del artículo, Tedesco discute la capacidad de las escuelas para responder a estas demandas. Cree que hay cuestiones pedagógicas que atender, sobre todo en relación a la formación de los docentes, no solamente en sus conocimientos, sino también en sus valores político-cívicos sobre la inclusión, y a los contenidos, que no pueden ser solamente adaptación a lo local, sino que tienen que promover 
experiencias de contacto con lo diferente, que enseñen la responsabilidad con respecto al otro. Señala el autor:

\begin{abstract}
La escuela puede cumplir un papel cultural y social significativo si asume un cierto grado de tensión y conflicto con la cultura externa a la institución. Su papel no es "adecuarse" a la cultura popular, ni tampoco, por supuesto, aislarse ni vaciarse de contenidos por la vía del empobrecimiento de los contenidos que ella transmite. Su papel es transmitir la capacidad de aprender a lo largo de toda la vida, la formación del núcleo básico del desarrollo cognitivo y valorativo desde el cual cada uno pueda elegir y definir su proyecto de vida. (Tedesco, 2004: 21)
\end{abstract}

Se ven, en esta enunciación, rastros de las primeras formulaciones sobre la escuela como espacio de ruptura con lo cotidiano y de formación en saberes que tienen un potencial transformador para las sociedades. A la par que el desplazamiento del lenguaje crítico radicalizado y de la preocupación con la dimensión regional, pueden verse continuidades entre los textos, por ejemplo, en la confianza en la educación como espacio público, de constitución, pero también de renovación de lo común. Y también se ve un hilo continuo de preocupación por la especificidad pedagógica y curricular de la enseñanza escolar, a la que otorga un valor que nunca, ni en el momento más álgido de crítica a la escuela en la década de 1970, dejó de percibir como significativa.

\title{
A modo de cierre
}

Los textos revisados muestran un modo de pensar la educación en diálogo con las ciencias sociales y la tradición intelectual crítica. Las referencias teóricas y las perspectivas disciplinarias cambian, lo cual podría decirse que no solo es esperable, sino también deseable. El seguimiento de las citas que hace Tedesco en estos artículos lo muestra como un lector curioso en varios idiomas, un intelectual que no deja de leer textos que provean hipótesis fuertes y de largo alcance sobre la sociedad y sus cambios. Si en la primera época sus citas fueron predominantemente argentinas y latinoamericanas, en los últimos años y probablemente como efecto de su paso por Ginebra, las referencias son a obras europeas y norteamericanas que piensan la condición contemporánea desde perspectivas mayoritariamente sociológicas y filosófico-políticas. También cambian los modos de enunciación: de intelectual crítico en la década de 1970 a intelectual que se piensa como hombre de Estado en las décadas siguientes, y finalmente, a partir del retiro de la Unesco, pasa a definirse como un intelectual cosmopolita que aporta lecturas sobre el porvenir del capitalismo y de la especie humana.

Estos desplazamientos pueden verse con claridad en uno de sus últimos trabajos, Educación y justicia social en América Latina, publicado en 2012. En este texto, Tedesco va a ser más explícito que en los trabajos anteriores sobre la necesidad de reformular el valor estratégico de la escuela para "los movimientos sociales" (2012: 147), los que ya no son nombrados como "movimiento obrero internacional" como en 1977, pero presumiblemente designan a sujetos sociales similares. Tedesco propone en esta obra revisar el legado del reproductivismo y la teoría crítica porque "subestimaron la importancia de la escuela en la ruptura del determinismo social del aprendizaje" (ibíd.) y en la conformación de una sociedad más justa. De alguna manera, puede decirse que está ajustando cuentas con su propio pasado, aun cuando nunca se hubiera enrolado en la crítica illichiana a la escuela. Considera que parte del legado de la crítica de la década de 1970 fue deslegitimar a la pedagogía escolar, y que eso dificultó la construcción de un sentido compartido sobre la educación. En implícita continuidad con lo dicho en el artículo de 1986, Tedesco sostiene que el consenso para acordar un núcleo común de contenidos curriculares es difícil de lograr no solamente por conflictos sociales también 
porque la propia teoría pedagógica ha desvalorizado la tarea de la escuela. A esto se suma el desafío de la tecnología: si antes el sentido pedagógico aparecía consistente con las estrategias pedagógicas, como en el normalismo, "hoy parece que los individuos pueden aprender por sí solos con la tecnología. [...] No hay dispositivo pedagógico claro para este nuevo momento" (2012: 218-219).

En este contexto, la escuela es, para Tedesco, el espacio mejor equipado para proveer esa formación del núcleo cognitivo que permita ubicarse de manera crítica frente a las transformaciones del capitalismo actual. El ideal es, para él, "una sociedad que garantice a todos sus miembros el goce de los derechos humanos básicos para una vida digna" (ibíd.: 220). Toma de Pierre Rosanvallon (en su libro La legitimidad democrática, 2009) la demanda de formar en tres figuras de legitimidad indispensables para la democracia: imparcialidad, reflexividad, proximidad. La formación en formas rigurosas del pensamiento científico y en el vivir juntos (el aprendizaje de la ciudadanía) aparecen como contenidos claves del núcleo común curricular que debe promover la escuela. Si bien no son propuestas nuevas, ya que estaban presentes en el texto de 1986, el argumento ahora incluye una reflexión sobre el nuevo capitalismo y la debilidad de los estados nacionales que suma una mayor complejidad a lo que antes se había identificado como aportes de los conocimientos escolares.

Puede verse en estas reflexiones una especie de cierre del círculo: antes y después, la escuela cumple una función política en la sociedad, como lo sostenía su admirado Sarmiento. Sin duda, los contenidos y formas de esa función fueron precisados por Tedesco de manera distinta a lo largo de su recorrido: si al inicio eran sobre todo el objeto de denuncia (la escuela como aparato ideológico dominante) y también de tibia búsqueda de alternativas, desde la década de 1980 fue clara su visión de la escuela como un dispositivo para crear otros futuros sociales, con modelos desarrollistas, como en 1986, o humanistas de nuevo cuño, como en el 2004 y 2012.

En este análisis de la trayectoria político-intelectual de Tedesco, puntuada por la historia reciente pero también por las particularidades de su propia biografía, puede verse el despliegue de un modo original de pensar la educación y particularmente la escuela, cuyas tareas y características fueron transformándose al correr de los procesos históricos de las últimas décadas. Tedesco combinó de manera singular una perspectiva histórica que le permitía mirar simultáneamente hacia atrás y hacia adelante, con una perspectiva interesada en comparar experiencias, modelos y propuestas en distintas latitudes afincada en las ciencias sociales y en los debates políticos de cada época. Esa mirada de largo plazo, inscripta en tradiciones específicas, pero también atenta a las transformaciones sociales y humanas en curso, y con cruces y referencias a distintas disciplinas, es la marca distintiva de sus aportes para pensar la educación. 


\section{Referencias bibliográficas}

» Altamirano, C., Piglia, R. y Sarlo, B. (dirs.). (1973). Editorial. En Los Libros, núm. 31, p. 3.

»Aranguren, M. (2010). La evolución de la revista Nueva Sociedad en el marco de la historia política y científico-social de América Latina (1972-1998), con foco en la llamada "Teoría de la Dependencia”. En Espacio Abierto, vol. 19, núm.1, pp. 5-25.

» Biasutto, C. [seudónimo de J. C. Tedesco] (1977). Educación y clase obrera. Ciudad de Mexico, Nueva Imagen.

»Braslavsky, C. (1982). Tendencias Centrales en la Política Educativa: unidad y diferenciación. En Revista Argentina de Educación, vol. 1, núm. 2, pp. 37-49.

"Ezpeleta, J., Teobaldo, M. y García, G. (1970). Cartas a una profesora. Educación, ideología y control social. En Los Libros, núm. 13, pp. 18-23.

» Gómez, S. (2016a). Juan Carlos Tedesco y el primer uso de Antonio Gramsci desde la teoría educativa argentina. En Propuesta Educativa, vol. 25, núm. 46, pp. 93-100.

»_. (2016b). El derrotero de Los Libros (1969-1976) y su crítica pedagógica. La interpretación educativa de Antonio Gramsci a través de Christine BuciGlucksmann. En Izquierdas, núm. 28, pp. 292-314.

"Gómez, S. y Kaplan, C. (2017). Itinerario político e intelectual de Juan Carlos Tedesco en los años sesenta y setenta: la modulación de una crítica pedagógica a través de la Revista de Ciencias de la Educación (1970-1975). En Revista de Historia de la Educación Argentina, vol. 18, núm. 1, pp. 24-40.

" Jaume, L. (2004). El pensamiento en acción: por otra historia de las ideas políticas. En Ayer vol. 53, núm. 1, pp. 109-130.

"Molloy, S. (1996). Acto de presencia. La escritura autobiográfica en Hispanoamérica. México, El Colegio de México.

"Palamidessi, M. y Suasnabar, C. (2007). Notas para una historia del campo de producción de conocimientos sobre educación en la Argentina. En Palamidessi, M.; Suasnabar, C. y Galarza, D. (comps.), Educación, Conocimiento y Política. Argentina 1983-2003, pp. 39-63. Buenos Aires, FLACSO-Manantial.

» Pulfer, D. (2017). Juan Carlos Tedesco (1944-2017). In memoriam. En Historia de la Educación Argentina, vol. 18, núm. 1, pp. 4-23.

"Rinesi, E. (2015). Populismo, democracia y “nueva izquierda” en América Latina. En Véliz, C. y Reano, A. (eds.), Gramáticas plebeyas. Populismo, democracia y nuevas izquierdas en América Latina, 23-51. Buenos Aires, UNGS-UNDAV.

» Spivak, G. Ch. (2017). Una educación estética en la era de la globalización. Ciudad de México, Siglo Veintiuno.

» Suasnábar, C. (2004). Universidad e intelectuales. Educación y política en la Argentina (1955-1976). Buenos Aires, FLACSO-Manantial.

»_. (2013). Intelectuales, exilios y educación: producción intelectual e innovaciones teóricas en educación durante la última dictadura. Rosario, Prohistoria. 
» Tedesco, J. C. (1973). Educación e ideología en Argentina. Notas para una investigación. En Los Libros, núm. 31, pp. 4-12.

» (1975). Educación y política en América Latina. En Los Libros, núm. 40, pp. 11-16.

"_. (1986). Crisis económica, educación y futuro en América Latina. En Revista Nueva Sociedad, núm. 84. En línea: <http://nuso.org/media/articles/ downloads/1413_1.pdf> (consulta: 12/12/2017).

»__ (2004). Incertidumbre y esperanza. Sobre la educación y el futuro. En Punto de Vista XXVII, núm. 8o, pp. 17-21.

》 . (2012). Educación y justicia social en América Latina. Buenos Aires, Fondo de Cultura Económica.

$\gg$ (2016). El Proyecto Educativo Autoritario en la obra de Cecilia Braslavsky. En Dussel, I. y Pineau, P. (eds.), Conocimiento, historia y política en la educación. El legado intelectual de Cecilia Braslavsky, 41-46. Buenos Aires, Santillana.

"Tedesco, J. C., Braslavsky, C. y Carciofi, R. (1983). El Proyecto Educativo Autoritario. Argentina 1976-1982. Buenos Aires, GEL-FLACSO.

\section{Bibliografía de consulta}

»Tedesco, J. C. (1973). Educación y clases sociales en la Argentina. Rosario, Centro de Estudios Sociales.

\section{Breve currículum de la autora}

\section{Inés Dussel}

Doctora en Educación, Universidad de Wisconsin-Madison; Magister en Ciencias Sociales, Facultad Latinoamericana de Ciencias Sociales (FLACSO) Argentina. Licenciada en Ciencias de la Educación, Universidad de Buenos Aires. Investigadora titular del Departamento de Investigaciones Educativas del Centro de Investigación y de Estudios Avanzados del Instituto Politécnico Nacional (DIE-CINVESTAV), México. Correo electrónico: idussel@gmail.com 